\title{
Multidimensional hydrodynamic simulations of the hydrogen injection flash
}

\author{
M. Mocák ${ }^{1}$, L. Siess ${ }^{1}$, and E. Müller ${ }^{2}$ \\ ${ }^{1}$ Institut d'Astronomie et d'Astrophysique, Université Libre de Bruxelles, ULB, CP 226, 1050 Brussels, Belgium \\ e-mail: [mmocak; siess] @astro.ulb.ac.be \\ 2 Max-Planck-Institut für Astrophysik, Postfach 1312, 85741 Garching, Germany \\ e-mail: ewald@mpa-garching.mpg.de
}

Received 22 March 2011 / Accepted 14 June 2011

\begin{abstract}
Context. The injection of hydrogen into the convection shell powered by helium burning during the core helium flash is commonly encountered during the evolution of metal-free and extremely metal-poor low-mass stars. Multidimensional hydrodynamic simulations indicate that the hydrogen injection may also occur in more metal-rich stars due to turbulent entrainment that accelerates the growth of the shell convection zone and increases its size. However, one-dimensional stellar models cast doubts that helium-flash hydrogen mixing does occur as it requires the crossing of an entropy barrier at the helium-hydrogen interface.

Aims. With specifically designed multidimensional hydrodynamic simulations, we aim to prove that an entropy barrier is no obstacle to the growth of the helium-burning shell convection zone in the helium core of a metal-rich Population I star, i.e. convection can penetrate into the hydrogen-rich layers for these stars, too. We study whether this is also possible in one-dimensional stellar evolutionary calculations.

Methods. We artificially shift the hydrogen-rich layer closer to the outer edge of the helium-burning shell convection zone in a Population I star with a mass of $1.25 M_{\odot}$, and simulate the subsequent evolution in two and three dimensions, respectively. We also perform stellar evolutionary calculations of the core helium flash in metal-rich stars implementing turbulent entrainment by means of a simple prescription. These simulations were performed with the Eulerian hydrodynamical code HERAKLES and the stellar evolution code STAREVOL, respectively.

Results. Our hydrodynamical simulations show that the helium-burning shell convection zone in the helium core moves across the entropy barrier and reaches the hydrogen-rich layers. This leads to a mixing of protons into the hotter layers of the core and to a rapid increase in the nuclear energy production at the upper edge of the helium-burning convection shell - the hydrogen injection flash. As a result, a second convection zone appears in the hydrogen-rich layers. In contrast to one-dimensional models, the entropy barrier separating the two convective shells from each other is largely permeable to chemical transport when allowing for multidimensional flow and consequently hydrogen is continuously mixed deep into the helium core. We find it difficult to replicate this behavior using one-dimensional stellar evolutionary calculations.
\end{abstract}

Key words. stars: evolution - hydrodynamics - convection

\section{Introduction}

The hydrogen injection flash is believed to commence during the core helium flash of metal-free and extremely metal-poor (metallicity $Z<10^{-4}$ ) low-mass stars when their helium core shell convection zone penetrates into the hydrogen-rich layers (Fujimoto et al. 1990; Hollowell et al. 1990; Schlattl et al. 2001; Cassisi et al. 2003; Weiss et al. 2004; Campbell \& Lattanzio 2008). Such an injection of protons is not expected for more metal-rich lowmass stars because the ignition of helium occurs further away from the hydrogen-rich layer and the entropy barrier between the helium and hydrogen layers is higher.

Multidimensional hydrodynamic simulations demonstrate that entropy barriers in stars are not as impenetrable as they at first appear to be. This is proven by mixing beyond the conventional Schwarzschild or Ledoux convection boundaries and the growth of convection zones on dynamical timescales caused by turbulent entrainment (Meakin \& Arnett 2007). Hydrodynamic simulations of shell convection during the core helium flash also suggest that convection could reach the outer hydrogen-rich layers of the star within a week ${ }^{1}$ at around the peak of the core helium flash even in metal-rich stars (Mocák et al. 2009, 2010). However in these models, the hydrogen-rich layers are still far away from the helium-burning shell convection zone preventing the simulation of the expected proton injection process within an affordable amount of computing time. Alternatively, one may study the evolution during the core helium flash up to a possible hydrogen injection using one-dimensional (hereafter 1D) stellar evolutionary calculations that include the effects of turbulent entrainment by means of a simple entrainment law. As these simulations had yet to be developed, we implemented in our 1D stellar evolution code a simple entrainment law based on

\footnotetext{
1 We find that the growth rate of the shell convection due to turbulent entrainment is on the order of a few meters per second for our hydrodynamic models, the exact value depending on the stability of the convection zone boundaries and the convective velocities near these boundaries (Meakin \& Arnett 2007). The last two quantities in turn depend not only on the dimensionality of the flow model (two- or threedimensional: hereafter 2D and 3D), but also on the spatial resolution of the simulation.
} 


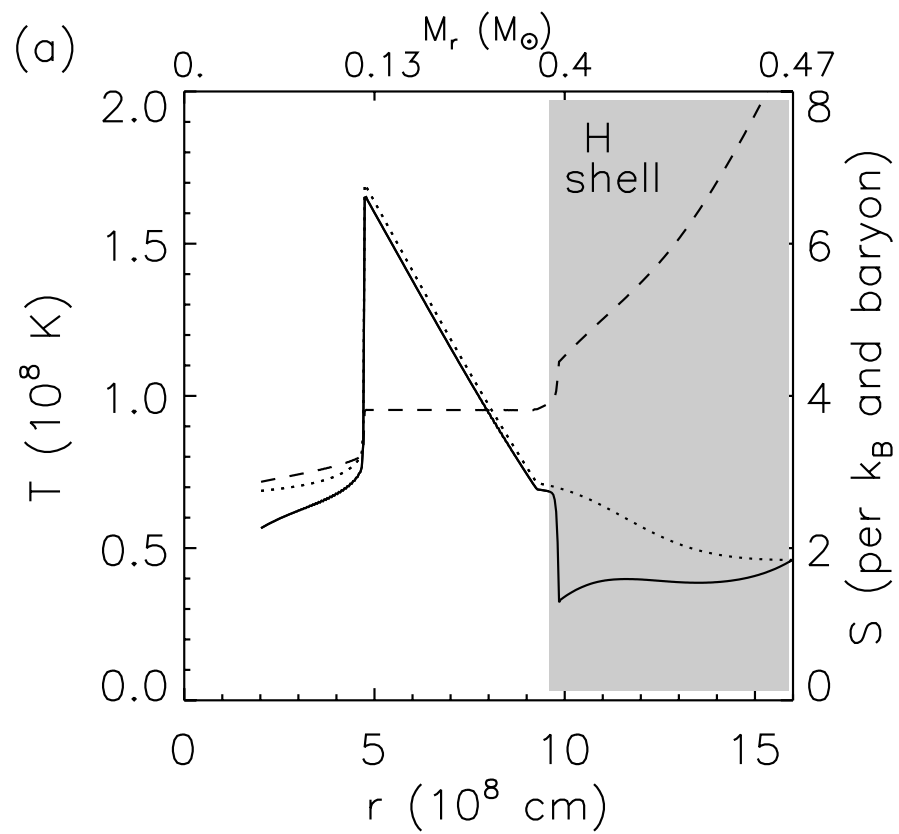

(b)

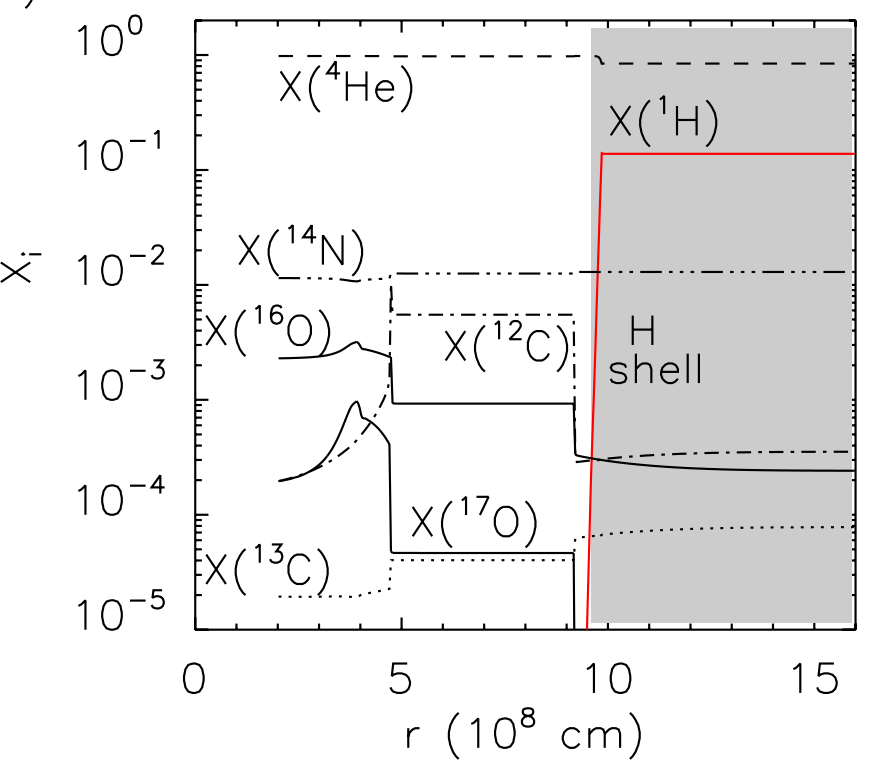

Fig. 1. a) Temperature distribution of the modified initial model (MOD, solid) and of the original model ( $M$, dotted) as a function of both radius $r$ and the enclosed mass $M_{r}$ in solar units $M_{\odot}$, respectively. The dashed curve gives the entropy profile of model MOD. b) Mass fraction of the main isotopes of model MOD as a function of radius. The hydrogen shell corresponds to the shaded region.

Table 1. Some properties of the modified initial stellar model MOD.

\begin{tabular}{ccccccc}
\hline \hline Model & $M$ & $M_{\mathrm{He}}$ & $R_{\mathrm{He}}$ & $L_{\mathrm{He}}$ & $L_{\mathrm{H}}^{p}$ & $L_{\mathrm{H}}^{f}$ \\
& {$\left[M_{\odot}\right]$} & {$\left[M_{\odot}\right]$} & {$\left[10^{8} \mathrm{~cm}\right]$} & {$\left[10^{9} L_{\odot}\right]$} & {$\left[10^{9} L_{\odot}\right]$} & {$\left[10^{9} L_{\odot}\right]$} \\
\hline MOD & 1.25 & 0.4 & 9.6 & 0.6 & 0.1 & 0.3 \\
\hline
\end{tabular}

Notes. Total mass $M$, mass $M_{\mathrm{He}}$, and radius $R_{\mathrm{He}}$ of the helium core, total nuclear energy production by helium burning $L_{\mathrm{He}}$, and by hydrogen burning $L_{\mathrm{H}}^{p}$ and $L_{\mathrm{H}}^{f}$, where the superscripts $p$ and $f$ denote that the respective values were obtained using a partial and full CNO network, respectively.

the ideas of Meakin \& Arnett (2007), and performed stellar evolutionary calculations of the core helium flash in low-mass Pop I stars. The entrainment rates adopted for these calculations are taken from the three-dimensional hydrodynamic simulations of Mocák et al. (2010).

Hydrodynamic simulations of similar phases of stellar evolution have also been carried out by Herwig et al. (2006, 2011). The results of their and our studies can have important implications for the hydrogen injection not only during the core helium flash but also during the early-AGB phase (i.e. the "dual shell flashes" described in Campbell \& Lattanzio (2008)) or in more advanced nuclear burning stages.

The paper is organized as follows. We introduce the numerical tools and the stellar input model used for our simulations in the next section. The hydrodynamic simulations of the hydrogen injection phase and the stellar evolutionary calculations mimicking turbulent entrainment during the core helium flash are presented in Sects. 3 and 4, respectively. A summary of our findings is given in Sect. 5 .

\section{Physical input and codes}

For the multidimensional hydrodynamic simulations we used the HERAKLES code (Mocák et al. 2008) derived from the

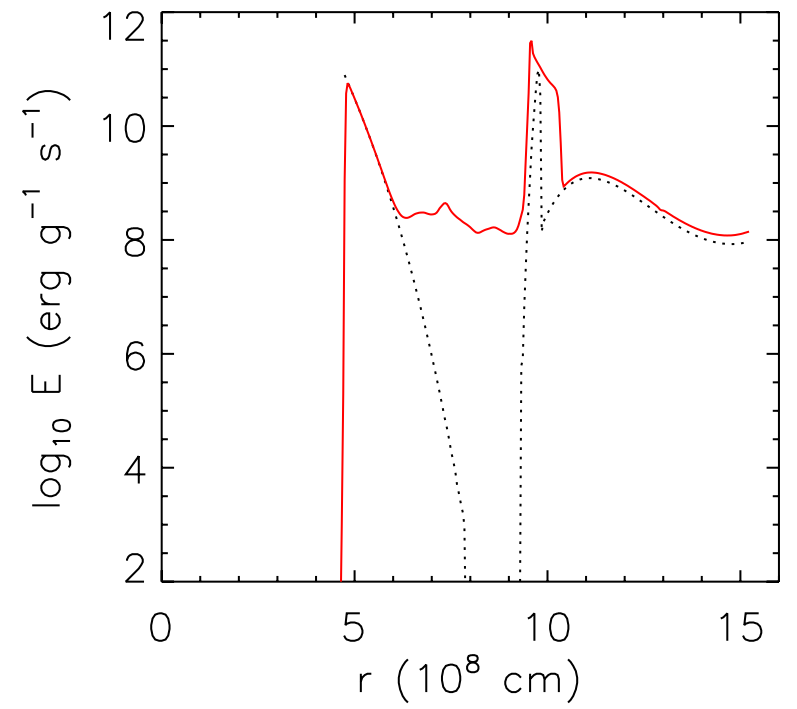

Fig. 2. Nuclear energy production rate as a function of radius $r$ for the 3D model exphif.3d.f at $t=0 \mathrm{~s}$ (dotted-black), and at $t=9800 \mathrm{~s}$ (solidred), respectively.

PROMETHEUS code (Fryxell et al. 1991; Müller et al. 1991). In brief, HERAKLES solves the Euler equations coupled with source terms describing self-gravity and nuclear burning. The hydrodynamic equations are integrated with the PPM reconstruction scheme (Colella \& Woodward 1984) and a Riemann solver for real gases according to Colella \& Glaz (1984). The evolution of the chemical species is described by a set of additional continuity equations (Plewa \& Müller 1999). Self-gravity is handled according to Müller \& Steimnetz (1995), gravitational potential being approximated by a $1 \mathrm{D}$ Newtonian potential obtained from the spherically averaged mass distribution. The code integrates nuclear networks with the semi-implicit BaderDeufelhard method (Bader \& Deuflhard 1983; Press et al. 1992). 
M. Mocák et al.: Multidimensional hydrodynamic simulations of the hydrogen injection flash

Table 2. Some properties of the $2 \mathrm{D}$ and $3 \mathrm{D}$ hydrodynamic simulations based on model MOD.

\begin{tabular}{lccccccccc}
\hline \hline Run & $w$ & $\begin{array}{c}\text { Grid } \\
\text { [name] }\end{array}$ & $\begin{array}{c}\Delta r \\
{\left[{ }^{\circ}\right]}\end{array}$ & $\begin{array}{c}\Delta r \times N_{\theta} \times N_{\phi} \\
{\left[10^{6} \mathrm{~cm}\right]}\end{array}$ & $\begin{array}{c}\Delta \phi \\
{\left[{ }^{\circ}\right]}\end{array}$ & $R_{\mathrm{e}}$ & $\left.\begin{array}{c}v_{\mathrm{c}} \\
{\left[10^{6}\right.}\end{array} \mathrm{cm} \mathrm{s}^{-1}\right]$ & $\begin{array}{c}\tau_{\text {conv }} \\
{[\mathrm{s}]}\end{array}$ & $\begin{array}{c}t_{\max } \\
{[\mathrm{s}]}\end{array}$ \\
\hline hifexp.3d.f & 45 & $370 \times 45 \times 45$ & 3.8 & 1 & 1 & $10^{2}$ & 1.0 & 1000 & 14400 \\
hifexp.2d.f & 90 & $370 \times 90$ & 3.8 & 1 & - & $10^{2}$ & 1.7 & 600 & 14400 \\
hifexp.2d.p & 90 & $370 \times 90$ & 3.8 & 1 & - & $10^{2}$ & 1.8 & 550 & 200000 \\
\hline
\end{tabular}

Notes. Wedge size $w$; number of grid points and resolution in the $r, \theta$, and $\phi$ direction, respectively; estimated Reynolds number $R_{\mathrm{e}}$; characteristic velocity $v_{\mathrm{c}}$ of the convective flow in helium-burning layers; typical convective turnover timescale $\tau_{\text {conv }}$ at time $t \sim 40000 \mathrm{~s}$ for model $2 \mathrm{~d}$.p and at $t \sim 2500 \mathrm{~s}$ for models 2 d.f and 3 d.f, respectively; $t_{\max }$ final evolutionary time.

As an initial model, we used the helium core structure of a $1.25 M_{\odot}, Z=Z_{\odot}$ star during the core helium flash at its peak nuclear luminosity (Table 1 ). In the initial model computed with the GARSTEC stellar evolution code (Weiss \& Schlattl 2000, 2007), the hydrogen-rich layers are artificially shifted closer to the outer boundary of the helium-burning shell convection zone at radius around $9.8 \times 10^{8} \mathrm{~cm}$. The lower boundary of the hydrogen layer is originally located at a radius of $1.9 \times 10^{9} \mathrm{~cm}$. The unmodified initial model (M) is described in more detail in Mocák et al. (2008, 2009). Compared to this model, the resulting hydrostatic structure of the modified initial model MOD differs by a temperature sink at the boundary between the helium- and hydrogen-rich layers (H-He boundary) at $9.5 \times 10^{8} \mathrm{~cm}<r<$ $10 \times 10^{8} \mathrm{~cm}$ (Fig. 1). At this boundary, the entropy increases almost discontinuously by $20 \%$, producing the so-called barrier. The temperature gradient in the helium-burning convection zone $\left(4.7 \times 10^{8} \mathrm{~cm}<r<9.2 \times 10^{8} \mathrm{~cm}\right)$ remains almost unaltered, as well as the nuclear energy production.

At the H-He boundary of model MOD around $r \sim 9.8 \times$ $10^{8} \mathrm{~cm}$, we find a narrow peak in the nuclear energy production rate (Fig. 2) that is due to some left-over hydrogen $(X \sim$ $10^{-4}$ ) when modifying the original initial model. In the layers near the $\mathrm{H}-\mathrm{He}$ boundary, the temperature is relatively high $\left(T \sim 5 \times 10^{7} \mathrm{~K}\right)$, and even if the hydrogen mass fraction is small, the nuclear energy production is significant. Although this feature is not seen in 1D stellar evolution simulations of the hydrogen injection flash (Hollowell et al. 1990), it may represent a somewhat more "realistic" situation, where the H-He interface is not a discontinuity but a moderately hot layer partially enriched with hydrogen.

For the stellar evolutionary calculations of the core helium flash, we implemented a simple turbulent entrainment law in the STAREVOL code (Siess 2006) and evolved a $1 M_{\odot}, Z=Z_{\odot}$ star for different entrainment rates guided by our 3D hydrodynamic simulations. To achieve hydrogen injection even in metalrich low-mass stars, we also tried the classical overshooting prescription based on the exponential decay of the convective velocity field beyond the convection zone boundaries (Freytag et al. 1996) with various efficiencies.

STAREVOL is a 1D Lagrangian stellar evolution code capable of computing the evolution of stars in the mass range $\left(0.1-60 M_{\odot}\right)$ from pre-main-sequence up to neon ignition. The nuclear network includes 53 species coupled by 177 nuclear reactions (Siess \& Arnould 2008), an accurate equation of state tested in the domain of very low-mass stars (Siess et al. 2000), and all relevant neutrino contributions (for a detailed description, the reader is referred to Siess (2006). This code has been widely used to study pre-main sequence stars (Siess et al. 1997), rotational mixing (Palacios et al. 2006), AGB nucleosynthesis (Siess et al. 2002), and super AGB stars (Siess 2010).

\section{Hydrodynamic simulations}

We performed two 2D (models hifexp.2d.f and hifexp.2d.p) and one 3D (model hifexp.3d.f) hydrodynamic simulation using different nuclear reaction networks. The last letter of the model name, $f$ and $p$, refers to simulations performed with a full and partial $\mathrm{CNO}$ reaction network (i.e. neglecting reactions related to ${ }^{13} \mathrm{~N}$ and ${ }^{15} \mathrm{O}$ ). These runs start from the modified initial model MOD with the shifted hydrogen profile. Some general properties of these simulations are summarized in Table 2, all of which were performed on an equidistant spherical grid ranging in radius from $2 \times 10^{8} \mathrm{~cm}$ to $1.6 \times 10^{9} \mathrm{~cm}$. The adopted grid resolution is motivated by our earlier work (Mocák et al. 2008), where we found convergence of the results starting at a radial grid resolution $\Delta r \sim 3.7 \times 10^{6} \mathrm{~cm}$ and an angular resolution of $\sim 1^{\circ}$. The boundary conditions in the radial direction are reflective, while periodic boundaries are imposed in the angular directions. For models hifexp.3d.f and hifexp.2d.f, the reaction network includes the species ${ }^{1} \mathrm{H},{ }^{3} \mathrm{He},{ }^{4} \mathrm{He},{ }^{12} \mathrm{C},{ }^{13} \mathrm{C},{ }^{13} \mathrm{~N},{ }^{14} \mathrm{~N},{ }^{15} \mathrm{~N},{ }^{15} \mathrm{O}$, ${ }^{16} \mathrm{O},{ }^{17} \mathrm{O},{ }^{20} \mathrm{Ne},{ }^{24} \mathrm{Mg}$, and ${ }^{28} \mathrm{Si}$, coupled by reactions of the full $\mathrm{CNO}$ cycle and the triple- $\alpha$ reaction. More details are given in the PhD thesis of Mocák (2009).

For the simulation hifexp.2d.p, we omitted the isotopes ${ }^{13} \mathrm{~N}$ and ${ }^{15} \mathrm{O}$, the $\beta$-decay reactions of the $\mathrm{CNO}$ cycle, and the ${ }^{12} \mathrm{C}(\mathrm{p}, \gamma){ }^{13} \mathrm{~N}$ reaction. Although this variant is unrealistic, it allows us to demonstrate that entropy barriers that are not sustained by sufficiently strong nuclear burning can be completely destroyed by the growing convection zone due to turbulent entrainment. When considering all species of the full CNO cycle, the nuclear energy production at the $\mathrm{H}-\mathrm{He}$ boundary is a factor of three higher than in the partial network and leads to the formation of a shell convection zone in the hydrogen-rich layers (Fig. 3; models hifexp.2d.f and hifexp.3d.f; see Sect. 3.1). No shell convection zone appears in the hydrogen-rich layers of the 2D model hifexp.2d.p.

\subsection{Simulations with the full CNO reaction network}

Our hydrodynamic 3D and 2D models, hifexp.3d.f and hifexp.2d.f (Fig. 3), are evolved with an accurate treatment of the $\mathrm{CNO}$ cycle on a numerical grid that has the same number of zones in both the radial and angular directions. This last feature allows us to compare the results of these simulations being biased only by the dimensionality of the simulation, but not by the grid resolution.

In the 3D (2D) model, hifexp.3d.f (hifexp.2d.f), after $800 \mathrm{~s}$ (1000s) convection fully develops in the layers located above the temperature maximum $T_{\max }\left(r \sim 4.7 \times 10^{8} \mathrm{~cm}\right)$. As soon as the first convective plumes reach the $\mathrm{H}$-He boundary, the dredgedown of hydrogen sets in. Up to $\sim 1500 \mathrm{~s}$, the composition profile in both models is similar, with a steep increase in the hydrogen mass fraction $X\left({ }^{1} \mathrm{H}\right)$ exceeding $10^{-9}$ just below the $\mathrm{H}-\mathrm{He}$ 

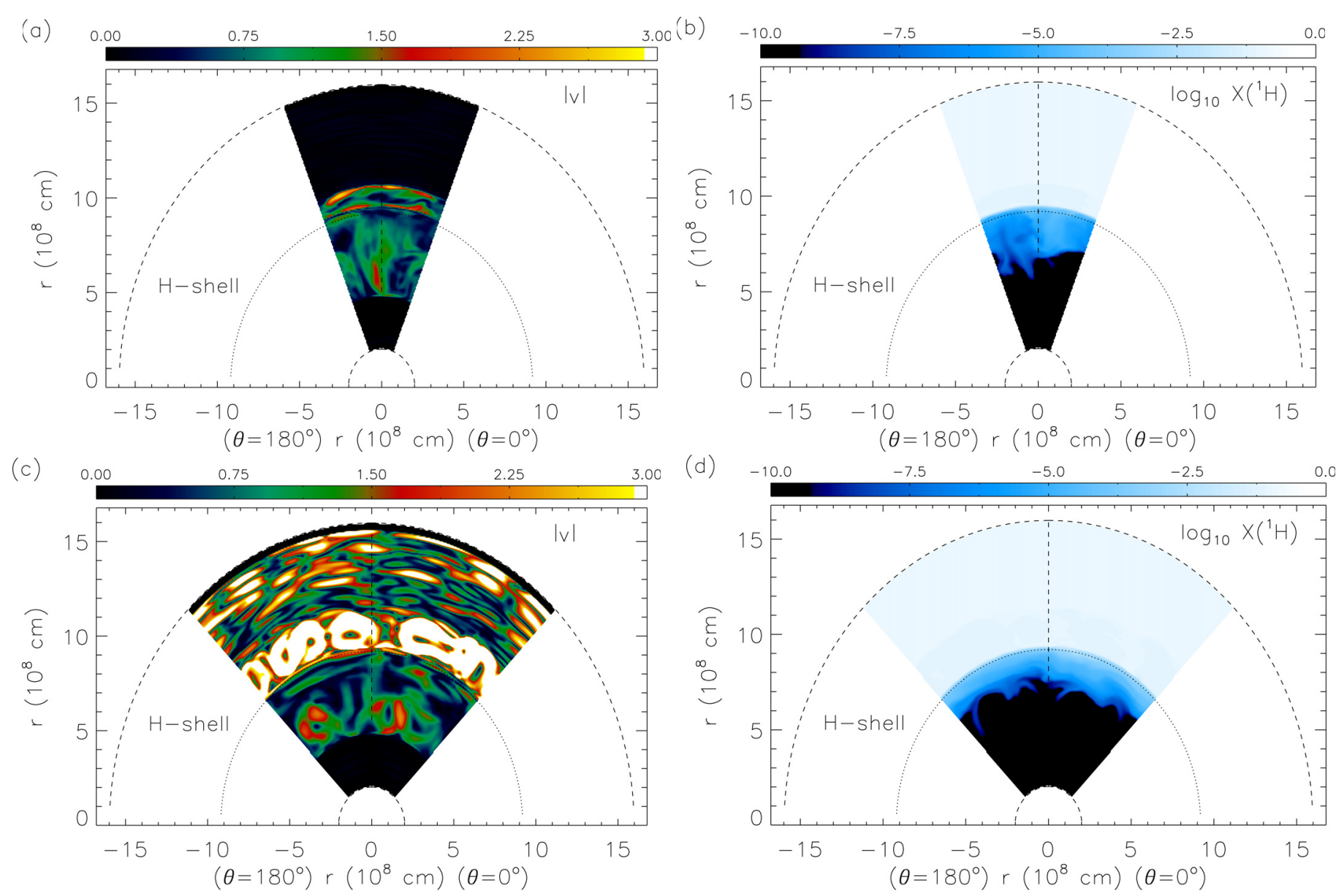

Fig. 3. Top row: snapshots showing contour plots of the modulus of the velocity $|v|$ (in units of $10^{6} \mathrm{~cm} \mathrm{~s}^{-1}$; left panel) and the hydrogen mass fraction (right panel) for the 3D model hifexp.3d.f at $t \sim 13300 \mathrm{~s}$ in the meridional plane $\left(\phi=0^{\circ}\right)$. Bottom row: same quantities but for the 2D model hifexp.2d.f. The dotted line separates helium- and hydrogen-rich layers (H-shell), and the dashed lines mark the boundaries of the computational domain.

interface (Fig. 4). Subsequently and until $t \sim 8000 \mathrm{~s}, X\left({ }^{1} \mathrm{H}\right)$ remains almost constant in the helium-burning convection zone of the 3D model, where $10^{-10}<X\left({ }^{1} \mathrm{H}\right)<10^{-8}$. This is in contrast to the $2 \mathrm{D}$ simulation, hifexp.2d.f, where $X\left({ }^{1} \mathrm{H}\right)$ continues to increase during this epoch. Between $8000 \mathrm{~s}$ and $12000 \mathrm{~s}$, the proton mass fraction increases in the helium-burning convection of both 2D and 3D models.

At $t \sim 12000 \mathrm{~s}$, the entropy barrier at the H-He interface in both models is steeper and almost twice as large as it was at the beginning of the simulations because of the enhanced nuclear burning. This increase in temperature and entropy at the interface slows down the injection of protons and the hydrogen mass fraction in the helium-rich layers decreases (Fig. 4).

As protons are dredged down into the helium core, they are captured by ${ }^{12} \mathrm{C}$ via the ${ }^{12} \mathrm{C}(\mathrm{p}, \gamma){ }^{13} \mathrm{~N}$ reaction, i.e. the hydrogen abundance decreases sharply with depth. Nevertheless, some protons can reach layers with temperatures exceeding $10^{8} \mathrm{~K}$, where their lifetime against the ${ }^{12} \mathrm{C}(\mathrm{p}, \gamma)^{13} \mathrm{~N}$ reaction is $\lesssim 100 \mathrm{~s}$ (Caughlan \& Fowler 1988). This is much shorter than their lifetime outside the He-burning convection shell. As the convective plumes have velocities of $10^{6} \mathrm{~cm} \mathrm{~s}^{-1}\left(2 \times 10^{6} \mathrm{~cm} \mathrm{~s}^{-1}\right)$ in the 3D (2D) model, hydrogen-rich gas is advected from the "colder" top of the He-burning convection shell to the aforementioned hot layers (located at $r \sim 7.7 \times 10^{8} \mathrm{~cm} \mathrm{~s}^{-1}$ ) in less then $200 \mathrm{~s}$ (100s). Our simulations show that this time is too short for the
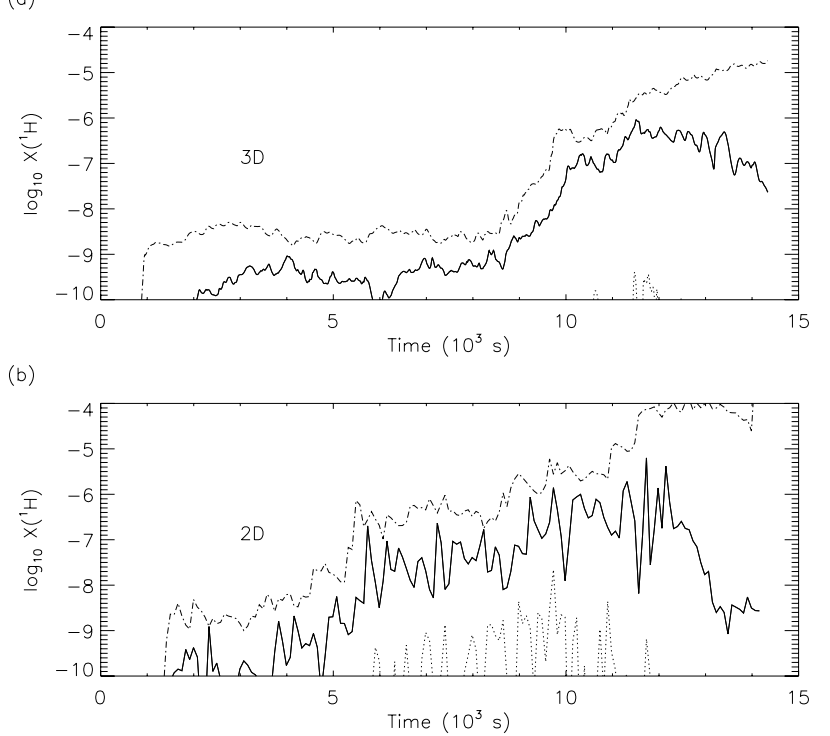

Fig. 4. Temporal evolution of the (logarithm of the) hydrogen mass fraction $\mathrm{X}\left({ }^{1} \mathrm{H}\right)$ at three different radii [temperatures] around the top, middle, and bottom of the helium-burning convection shell, respectively: $r_{1}=8.8 \times 10^{8} \mathrm{~cm}\left[T_{1} \sim 0.8 \times 10^{8} \mathrm{~K}\right]$ (dash-dotted), $r_{2}=7.1 \times 10^{8} \mathrm{~cm}$ $\left[T_{2} \sim 1.2 \times 10^{8} \mathrm{~K}\right]$ (solid), and $r_{3}=5.4 \times 10^{8} \mathrm{~cm}\left[T_{3} \sim 1.5 \times 10^{8} \mathrm{~K}\right]$ (dotted). The upper panel (a) gives the results for the 3D model hifexp.3d.f, and the lower panel (b) for the 2D model hifexp2d.f, respectively. 
(a)

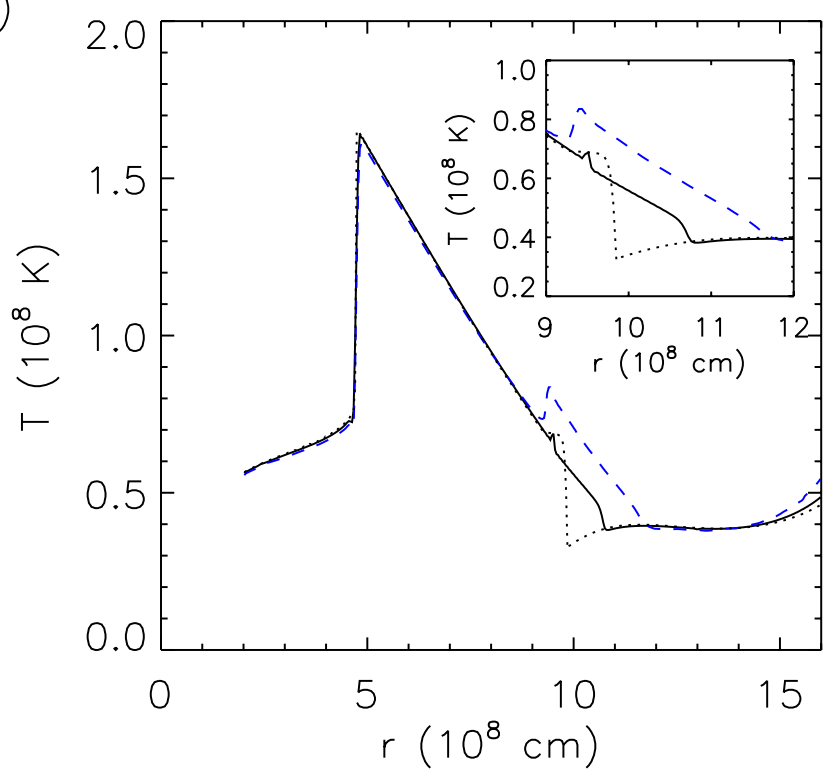

(b)

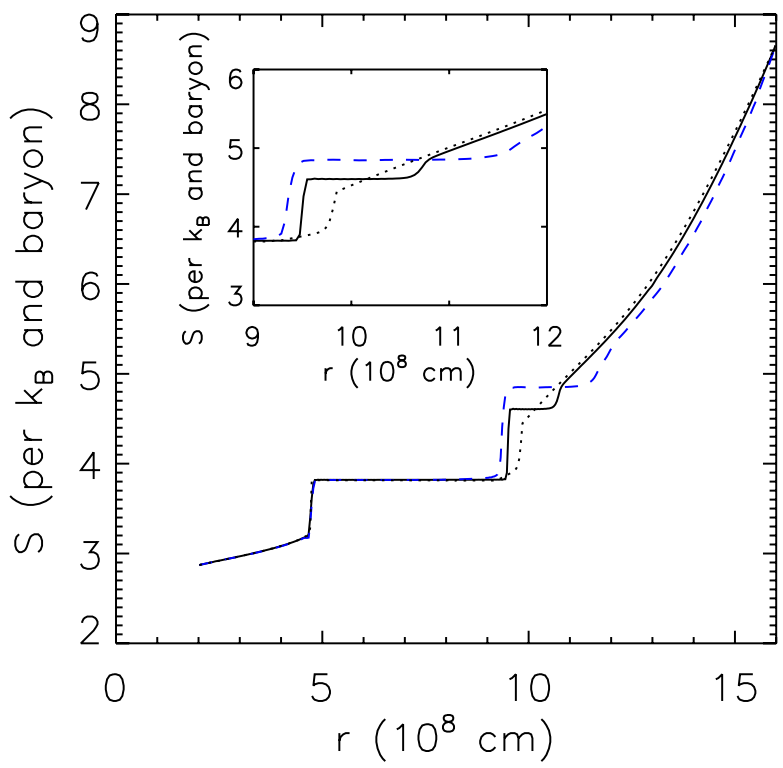

Fig. 5. Radial distribution of a) temperature and b) entropy at $t=0 \mathrm{~s}$ (dotted) and $t_{1}=13500 \mathrm{~s}$ for the 2D model hifexp.2d.f (dashed, blue), and the 3D model hifexp.3d.f (solid), respectively. The inserts show a zoom of the region around the $\mathrm{H}-\mathrm{He}$ interface.

hydrogen to burn completely while being advected to layers with temperatures $\gtrsim 10^{8} \mathrm{~K}$ (Fig. 4).

The differences between the $X\left({ }^{1} \mathrm{H}\right)$ distributions of the 2D and $3 \mathrm{D}$ model can be understood by realizing that the convective velocities in the 3D model are almost a factor of two smaller than in the $2 \mathrm{D}$ one. Hence, there is more time for hydrogen to burn during its transport in the 3D model than in the $2 \mathrm{D}$ one, i.e. hydrogen is able to reach the hot layers in larger amounts in the latter case (Fig. 4). Besides higher convective velocities, the convective plumes arising at the $\mathrm{H}-\mathrm{He}$ boundary also fill a larger volume fraction in the 2D model compared with the 3D one, where the plumes are smaller and narrower.

The nuclear energy production at the H-He boundary and the impacting convective plumes change the initial properties of the $\mathrm{H}-\mathrm{He}$ interface. At the start of the simulations, the boundary is characterized by a steep radial step in both the temperature and entropy profiles. However, when convection reaches the interface, the single step structure of the temperature and entropy profiles evolves into a two-step structure exhibiting a narrow entropy plateau between the steps (Fig. 5). This plateau reflects the formation of a secondary convection shell, located in the hydrogen-rich layers and powered by the $\mathrm{CNO}$ cycle. We find higher absolute values of both the entropy and temperature in the 2D model, hifexp.2d.f, compared to its 3D counterpart across the forming new convection shell. This can be explained by the stronger nuclear burning occurring in that model, which also leads to a faster growth of the hydrogen-burning convection shell.

Nuclear burning at the $\mathrm{H}-\mathrm{He}$ interface steadily increases during both simulations, and eventually leads to the formation of a distinct second temperature peak (Figs. 5, 7). This behavior is well known from 1D stellar evolutionary calculations of the core helium flash in extremely metal-poor stars, where the heliumburning convection shell splits after the injection of hydrogen. However, the splitting process seen in 1D stellar calculations differs from that found in our multidimensional simulations. In 1D simulations, the splitting occurs because of temperature peak in the convective helium-burning shell at the location where hydrogen burning by the ${ }^{12} \mathrm{C}(\mathrm{p}, \gamma)^{13} \mathrm{~N}$ reaction is proceeding the fastest.

In our hydrodynamic models, the appearance of a second temperature peak is preceded by a slow migration of the $\mathrm{H}-\mathrm{He}$ boundary into the helium-burning convective shell, which forces it to retreat slightly (Fig. 7). Eventually, when the nuclear energy release and the temperature at the $\mathrm{H}-\mathrm{He}$ boundary become sufficiently high, we observe the appearance of a new convection shell in the hydrogen-rich layers above the original one. Hence, what actually happens is not a splitting, but a "retreat" of the initial helium-burning convection shell and the birth of a new one on top of it powered by the burning of hydrogen.

Our hydrodynamic simulations exhibit another major distinct property: the absence of an impermeable radiative layer between the two convective shells. The mixing of nuclear species between these layers is not prohibited, but occurs during the whole event (Fig. 6) with decreasing efficiency towards the end of simulation.

The evolution of the hydrodynamic models is likely representative of early phases during the dual core and He-shell flashes, and indicates that the entropy barrier does not inhibit hydrogen dredge-down. However, this conclusion should be moderated by the finding that the entropy at the $\mathrm{H}-\mathrm{He}$ boundary can be increased by the rise of the nuclear energy production. Eventually, this entropy barrier may become so high that mixing ceases completely. What we have shown here is that, given our initial conditions, nothing seems to prevent $\mathrm{H}$-injection, at least in the early stages. A similar behavior was also found by Herwig et al. (2011) in their 3D simulations of the hydrogen injection into the helium-shell-flash convection zone, which helped them to explain major features observed in the spectra of Sakurai's object (Duerbeck et al. 2000) (a post-AGB object experiencing its last helium flash). We finally note that proton injection may have interesting consequences for the production of s-process elements as some ${ }^{13} \mathrm{C}$ can be produced, which is a source of neutrons (Campbell et al. 2010).

We note that during its final evolution stage, the 2D model hifexp.2d.f eventually experiences another unrealistic nuclear runaway when $\mathrm{He}$ ignites in the secondary convection shell because of the high temperature $\gtrsim 10^{8} \mathrm{~K}$ at the $\mathrm{H}-\mathrm{He}$ boundary; the shell then rapidly expands to the outer boundary of the computational domain, when we stopped the calculation (Fig. 6b). 

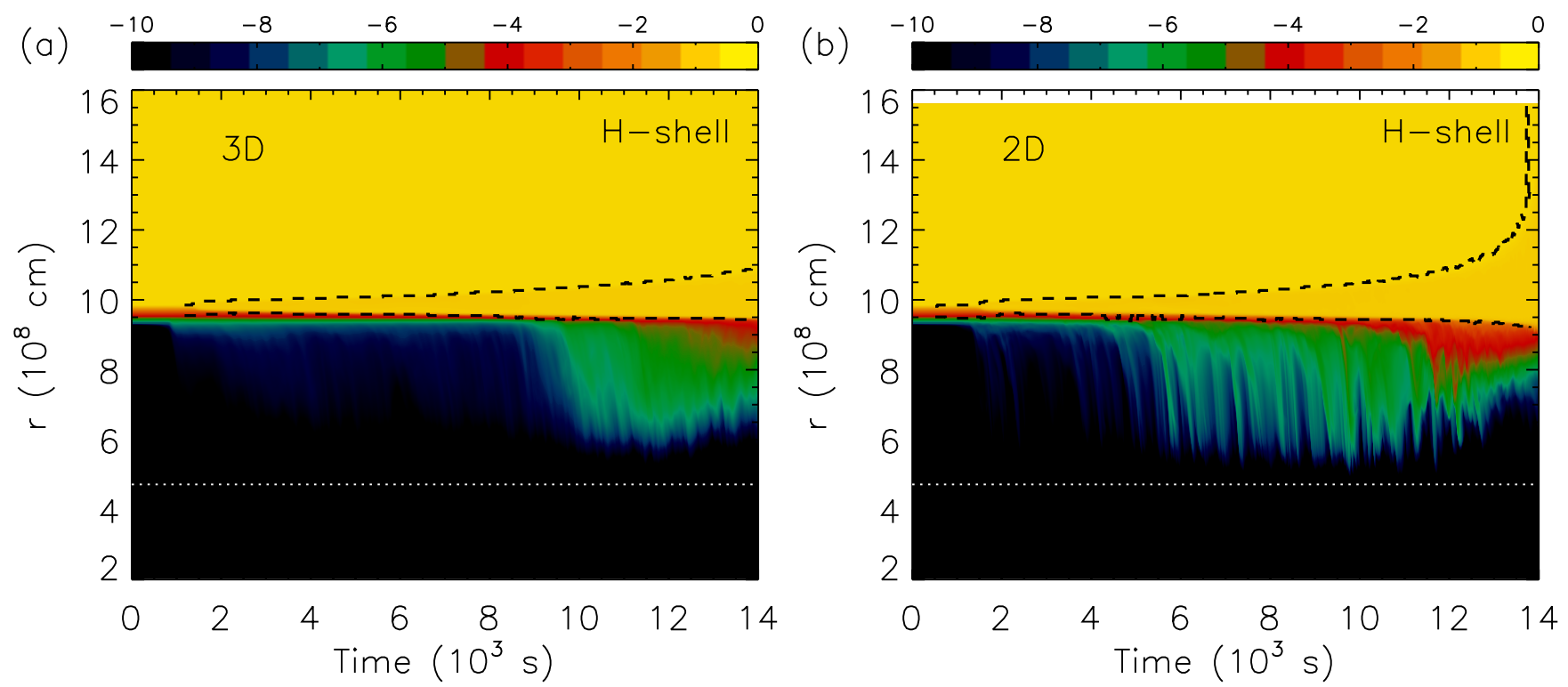

Fig. 6. a) Temporal evolution of the angle-averaged radial distribution of (the logarithm of) the hydrogen mass fraction for the 3D model hifexp.3d.f, and b) for the 2D model hifexp.2d.f, respectively. The dashed lines delineate the boundaries of the newly forming hydrogen-burning convection shell, and the dotted line marks the bottom of the helium-burning convection zone.

(a)

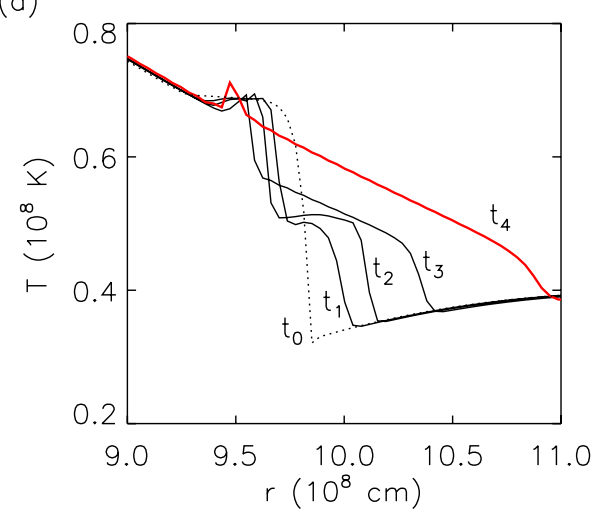

(b)

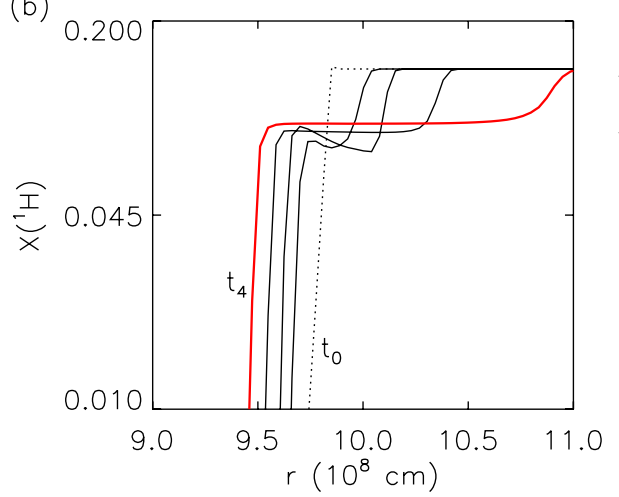

(c)

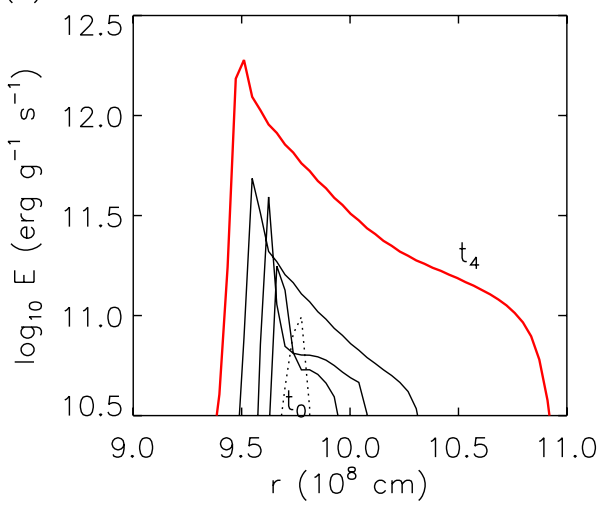

Fig. 7. Radial distribution of a) temperature, $\mathbf{b}$ ) hydrogen mass fraction, and c) energy production rate at the H-He interface for the 3D model hifexp.3d.f at five different times: $t_{0}=0 \mathrm{~s}, t_{1}=3230 \mathrm{~s}, t_{2}=6500 \mathrm{~s}, t_{3}=10400 \mathrm{~s}$, and $t_{4}=14340 \mathrm{~s}$.

Concerning this unexpected behavior we have to emphasize that these hydrodynamical simulations are strongly dependent on the initial conditions. In particular, we note that in the initial model MOD the layers at the H-He boundary are highly degenerate (with a degeneracy parameter $\psi \approx 10)^{2}$. This large degeneracy favors a nuclear runaway. In addition, our multidimensional simulations are likely to underestimate the star's expansion and subsequent cooling because of the reflective boundary conditions imposed in the radial direction. These numerical restrictions limit the cooling and favor the aforementioned helium runaway.

\subsection{Simulation with partial CNO network}

In the 2D simulation hifexp.2d.p, we considered neither the $\beta$ decay reactions ${ }^{13} \mathrm{~N}\left(\beta^{-}\right){ }^{13} \mathrm{C}$ and ${ }^{15} \mathrm{O}\left(\beta^{-}\right){ }^{15} \mathrm{~N}$ nor the ${ }^{12} \mathrm{C}(\mathrm{p}, \gamma){ }^{13} \mathrm{~N}$ reaction, this neglect leading to a reduced energy production rate

\footnotetext{
${ }^{2}$ Matter at the H-He boundary after proton injection (and some earlier expansion) in a 1D Population III model of $0.85 M_{\odot}$ computed by Simon Campbell (private communication) (Mocák 2009) has a degeneracy parameter $\psi \approx-2$.
}

by roughly a factor of three at the $\mathrm{H}-\mathrm{He}$ interface. We were then able to demonstrate, that entropy barriers at convection zone boundaries that are insufficiently sustained by nuclear burning are penetrable and unable to prevent the growth of the convection zone because of turbulent entrainment on dynamic timescales. By neglecting proton capture on ${ }^{12} \mathrm{C}$ in the simulation, we obtain an incorrect hydrogen profile and a reduced nuclear energy production rate. This result explains why we do not observe here the formation of the secondary hydrogen-burning shell convection produced in our hydrodynamic models with the full nuclear CNO reaction network (see previous subsection).

In this model, helium-burning convection starts at $t \sim 1000 \mathrm{~s}$ and extends throughout the entirety of the initially convectively unstable shell as determined by the Schwarzschild criterion. The convection shell is located between the radius of the temperature maximum and the base of the hydrogen-rich layers. It is characterized by vortices, which are typical structures of 2D simulations caused by the imposed axial symmetry. Their width is equal to the radial extent of the convection shell, i.e. $r \sim$ $5 \times 10^{8} \mathrm{~cm}$, which is also the width of the convection zone in the unmodified initial model (M). 
The shell convection reaches the hydrogen-rich layers shortly after the appearance of the first convective plumes. It initiates the dredge-down of protons deep into the helium core, and causes the outer boundary of the convection shell to cross the entropy barrier at the H-He interface (Fig. 8). The value of the entropy at this interface constantly decreases because of turbulent entrainment, which effectively shifts the entropy barrier to larger radii.

The entrainment rate of the outer convection boundary is initially relatively high, around $70 \mathrm{~m} \mathrm{~s}^{-1}$, but after roughly $30000 \mathrm{~s}$ drops and eventually relaxes to an almost constant value of $\sim 4.5 \mathrm{~m} \mathrm{~s}^{-1}$. The entropy barrier has a stabilizing effect because it decreases the entrainment velocity. However, it does not prevent the advance of the convection zone into the H-rich layers and leads to an erosion of the entropy gradient. After $170000 \mathrm{~s}$, the shell convection has moved $\sim 8 \times 10^{7} \mathrm{~cm}$ into the H-rich buffer, meaning that almost $12 \%$ of the hydrogen-rich shell included in the simulation (limited by the outer radial grid boundary at $1.6 \times 10^{9} \mathrm{~cm}$ ) is already entrained within $47 \mathrm{~h}$, or $\sim 300$ convective turnover timescales.

In this simulation, protons are able to reach layers with temperatures as high as $\sim 1.8 \times 10^{8} \mathrm{~K}$. However, such a deep mixing of hydrogen into the helium burning layers is not realistic because protons should have been captured by ${ }^{12} \mathrm{C}$ at an earlier stage.

\section{Stellar evolutionary calculations}

To mimic the effects of the turbulent entrainment in 1D stellar evolutionary calculations, we experiment and implement a simple entrainment law

$\dot{M}_{\mathrm{E}}=\frac{\partial M}{\partial r} u_{\mathrm{E}}$

where $M$ is the mass of the shell at radius $r$ and $u_{\mathrm{E}}$ is the entrainment rate (Meakin \& Arnett 2007). We simulated a $1 M_{\odot}, Z=Z_{\odot}$ star up to the core helium flash. At first, we use an entrainment rate of $6.7 \times 10^{2} \mathrm{~cm} \mathrm{~s}^{-1}$ as found by Mocák et al. (2009) in their 3D hydrodynamic simulations of the core helium flash. The entrainment is switched on only shortly before the flash reaches its maximum intensity (maximum nuclear energy generated in the helium core). We find that the He-convection zone boundary is unable to cross the entropy barrier and penetrate into the hydrogen shell. This result also holds when increasing the entrainment rate by one or even two orders of magnitude, and is partially due to the inherent time dependence of the mixing prescription.

For a given timestep $\Delta t$, mixing will indeed extend over a mass range given by $\Delta M_{\mathrm{e}}=\dot{M}_{\mathrm{E}} \Delta t$. Practically, we simply shift the convective boundary to the new mass location $M_{\text {conv }}^{\text {edge }}+\Delta M_{\mathrm{e}}$. As the helium convection shell approaches the H-rich layer, the timestep, which is constrained among other things by the rate of change of the nuclear luminosity, decreases thus preventing the injection of protons and a subsequent $\mathrm{H}$-flash. Eventually, a (small) timestep is found that allows the simulation to proceed and after some time, the helium-shell convection zone naturally quenches and $\mathrm{H}$-injection is avoided.

On the other hand, if we experiment with overshooting by using the exponential decay of the convection velocity in the radiative layers, mixing is treated as a diffusive process with the diffusion coefficient given by

$D_{\text {over }}=D_{\text {conv }} \exp \left(-\left|r-r_{\text {edge }}\right| /\left(2 f H_{\mathrm{P}}\right)\right.$

where $H_{\mathrm{P}}$ is the pressure scale-height at the edge of the convective zone and $f$ a free parameter. This treatment has two main (a)

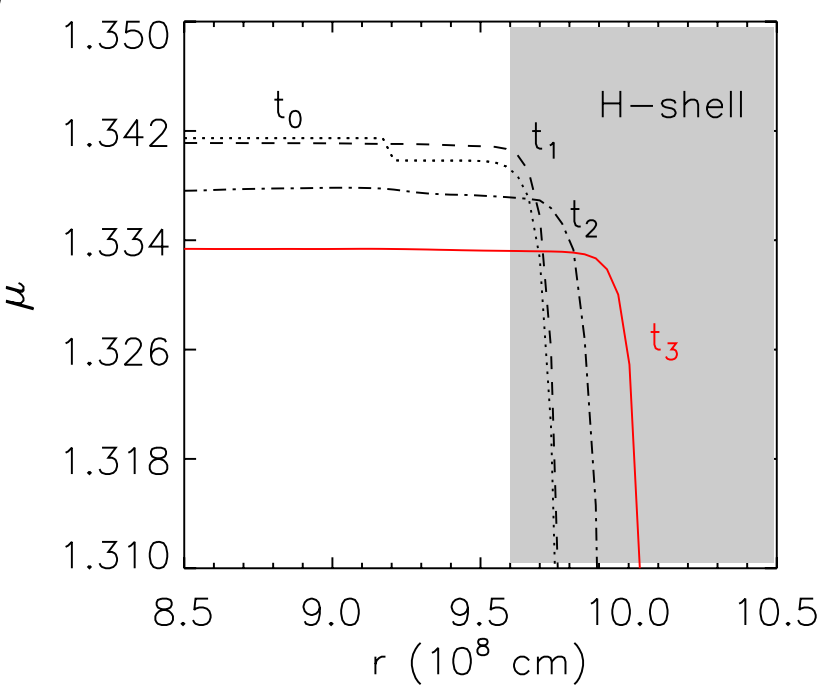

(b)

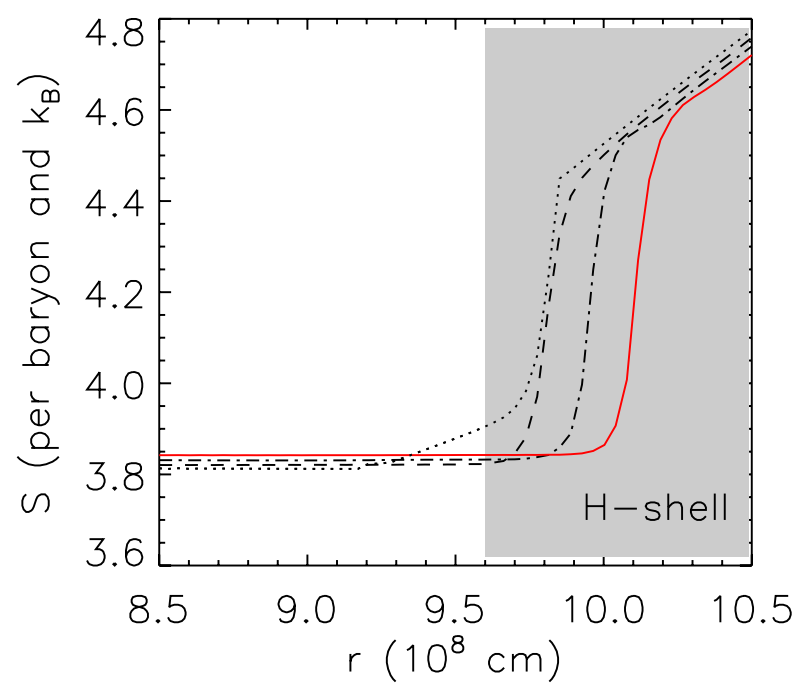

Fig. 8. Mean molecular weight a) and entropy b) at the outer edge of the convection zone of the $2 \mathrm{D}$ model simulated with the partial CNO network (hifexp.2d.p) at four different times: $t_{0}=0 \mathrm{~s}$ (dotted), $t_{1}=$ $46600 \mathrm{~s}$ (dashed), $t_{2}=94200 \mathrm{~s}$ (dash-dotted), and $t_{3}=142000 \mathrm{~s}$ ( red).

differences from the previous prescription: mixing is treated as a diffusive process so the penetration of the He-shell convection is "smoother" and the new convective boundary at $t+\Delta t$ does not explicitly depend on its location at time $t$ and on the timestep. Our 1D experiments indicate that at solar metallicity, a substantial overshooting is required to trigger an injection with $f \sim 1$. This injection then leads to the splitting of the He-shell convection and after some structural readjustment to the pollution of the envelope. We report $\mathrm{C}$ and $\mathrm{N}$ enrichments by a factor of 2 or 3 . Interestingly, we find that after this mixing event, the ${ }^{12} \mathrm{C} /{ }^{13} \mathrm{C}$ ratio has dropped to below $\sim 10$. Moreover, these He-core burning stars are carbon-rich $(\mathrm{C} / \mathrm{O} \sim 1.7)$ and have high ${ }^{7} \mathrm{Li}$ abundances $(\varepsilon(\mathrm{Li}) \approx 3.5)$. Some of these characteristics are found in J-stars but in conflict with observations, our models predict too large ${ }^{16} \mathrm{O} /{ }^{17} \mathrm{O}$ ratio (by a factor of at least two) and the $\mathrm{C} / \mathrm{N}$ ratio is too low in our models. 


\section{Summary}

We have performed hydrodynamic simulations of shell convection in the core of a low mass star at the peak luminosity of the helium flash. In these computations, the hydrogen-rich layers were artificially shifted down into the helium core. They have allowed us to demonstrate a penetration of the helium-burning shell convection into the hydrogen layers caused by turbulent entrainment, despite the presence of an entropy barrier between them. The consequences of this result are the following.

Hydrodynamic models computed with the full CNO reaction network leads to the formation of a secondary shell convection zone in the hydrogen-rich layers. This zone is powered by hydrogen burning and is located above the already-present heliumburning shell convection. These simulations show (in contrast to 1D stellar calculations of similar evolutionary phases) that the mixing of protons down to the helium-burning convection shell is not completely inhibited, although it eventually slows down because of an increase in the nuclear energy production and the growth of the entropy barrier at the interface between the heliumburning and hydrogen-burning convection shell.

In our hydrodynamic model computed with a reduced nuclear reaction network, the energy production rate in the hydrogen-rich layers is smaller and we do not detect the appearance of the hydrogen-burning convection shell. This simulation shows that an entropy barrier insufficiently sustained by nuclear burning does not act as an obstacle to the growing helium-burning convection shell due to turbulent entrainment. The entrainment rate of the outer rim of the helium-burning convection zone reaches a roughly constant non-vanishing value.

Given our initial model of a low-mass and metal-rich star, the aforementioned situations were difficult to reconstruct using a one-dimensional stellar evolution code.

Acknowledgements. The simulations were performed at the Rechenzentrum Garching on the IBM Power6 system. The authors want to thank Frank Timmes for some of his publicly available FORTRAN subroutines which we used in the HERAKLES code. Miroslav Mocák acknowledges financial support from the Communauté française de Belgique - Actions de Recherche Concertées. L.S. is
FNRS research associate. We also thank John Lattanzio for summarizing the observations about J-stars.

\section{References}

Bader, G., \& Deuflhard, P. 1983, Numer. Math., 41, 373

Campbell, S. W., \& Lattanzio, J. C. 2008, A\&A, 490, 769

Campbell, S. W., Lugaro, M., \& Karakas, A. I. 2010, A\&A, 522, L6

Cassisi, S., Schlattl, H., Salaris, M., \& Weiss, A. 2003, ApJ, 582, L43

Caughlan, G. R., \& Fowler, W. A. 1988, Atomic Data and Nuclear Data Tables, 40, 283

Colella, P., \& Glaz, H. H. 1984, J. Comput. Phys., 59, 264

Colella, P., \& Woodward, P. R. 1984, J. Comput. Phys., 54, 174

Duerbeck, H. W., Liller, W., Sterken, C., et al. 2000, AJ, 119, 2360

Freytag, B., Ludwig, H., \& Steffen, M. 1996, A\&A, 313, 497

Fryxell, B., Arnett, D., \& Müller, E. 1991, ApJ, 367, 619

Fujimoto, M. Y., Iben, I. J., \& Hollowell, D. 1990, ApJ, 349, 580

Herwig, F., Freytag, B., Hueckstaedt, R. M., \& Timmes, F. X. 2006, ApJ, 642, 1057

Herwig, F., Pignatari, M., Woodward, P. R., et al. 2011, ApJ, 727, 89

Hollowell, D., Iben, I. J., \& Fujimoto, M. Y. 1990, ApJ, 351, 245

Meakin, C. A., \& Arnett, D. 2007, ApJ, 667, 448

Mocák, M. 2009, Ph.D. Thesis, Max-Planck-Institut für Astrophysik, Garching bei München

Mocák, M., Müller, E., Weiss, A., \& Kifonidis, K. 2008, A\&A, 490, 265

Mocák, M., Müller, E., Weiss, A., \& Kifonidis, K. 2009, A\&A, 501, 659

Mocák, M., Campbell, S. W., Müller, E., \& Kifonidis, K. 2010, A\&A, 520, A114

Müller, E., Fryxell, B., \& Arnett, D. 1991, in ESO/EIPC Workshop on Supernova 1987A and other Supernovae, 99, 116

Müller, E., \& Steimnetz, M. 1995, Comp. Phys. Commun., 89, 45

Palacios, A., Charbonnel, C., Talon, S., \& Siess, L. 2006, A\&A, 453, 261

Plewa, T., \& Müller, E. 1999, A\&A, 342, 179

Press, W. H., et al. 1992, in Numerical Recipes in FORTRAN, The Art of Scientific Computing, 2nd edn. (Cambridge: Cambridge University Press), 1

Schlattl, H., Cassisi, S., Salaris, M., \& Weiss, A. 2001, ApJ, 559, 1082

Siess, L. 2006, A\&A, 448, 717

Siess, L. 2010, A\&A, 512, A10

Siess, L., \& Arnould, M. 2008, A\&A, 489, 395

Siess, L., Forestini, M., \& Dougados, C. 1997, A\&A, 324, 556

Siess, L., Dufour, E., \& Forestini, M. 2000, A\&A, 358, 593

Siess, L., Livio, M., \& Lattanzio, J. 2002, ApJ, 570, 329

Weiss, A., \& Schlattl, H. 2000, A\&AS, 144, 487

Weiss, A., Schlattl, H., Salaris, M., \& Cassisi, S. 2004, A\&A, 422, 217

Weiss, A., \& Schlattl, H. 2007, Ap\&SS, 341 